\title{
Chemically specific imaging and in-situ chemical analysis of articular cartilage with stimulated Raman scattering
}

\author{
Jessica Mansfield ${ }^{*}, 1$, Julian Moger ${ }^{1}$, Ellen Green ${ }^{1}$, Charlotte Moger ${ }^{2}$ and C Peter Winlove ${ }^{1}$ \\ ${ }^{1}$ Physics, University of Exeter, Stocker Rd, Exeter, EX4 4QL \\ 2 Research Knowledge Transfer, University of Exeter, Rennes Drive, Exeter, EX4 4RN
}

Key words: Stimulated Raman Scattering, Coherent anti-stokes Raman scattering, second harmonic generation, two-photon fluorescence, spontaneous Raman, cartilage, mineralised tissue

\begin{abstract}
Stimulated Raman Scattering has been applied to unstained samples of articular cartilage enabling the investigation of living cells within fresh tissue. Hyperspectral SRS measurements over the $\mathrm{CH}$ vibrational region showed variations in protein and lipid content within the cells, pericellular matrix and interteritorial matrix. Changes in the cells and pericellular matrix were investigated as a function of depth into the cartilage. Lipid was detected in the pericellular matrix of superficial zone chondrocytes. The spectral profile of lipid droplets within the chondrocytes indicated that they contained predominantly unsaturated lipids. The mineral content has been imaged by using the $\mathrm{PO}_{4}{ }^{3-}$ vibration at $959 \mathrm{~cm}^{-1}$ and the $\mathrm{CO}_{3}{ }^{2-}$ vibration at $1070 \mathrm{~cm}^{-1}$. Both changes in cells and mineralization are known to be important factors in the progression of osteoarthritis. SRS enables these to be visualised in fresh unstained tissue and consequently should benefit osteoarthiritis research
\end{abstract}

\section{Introduction}

Articular cartilage is the connective tissue covering the ends of the bones within the synovial joint and provides a low-friction bearing and shock absorber. Failure of the cartilage gives rise to osteoarthritis, which is a widespread and debilitating condition, whose development is still imperfectly understood.

Cartilage contains few cells and the extracellular matrix comprises approximately $70 \%$ water, $20 \%$ type II collagen $8 \%$ proteoglycans and $2 \%$ other proteins [1]. The organisation of the matrix had been explored extensively using light and electron microscopy, generally with the aid of classical histochemical stains or antibody labelling. Four distinct zones can be identified in the articular cartilage as illustrated in figure 1 . The present study is concerned particually with the zone of calcified cartilage and the pericellular matrix which both pose particular problems in conventional histology.

The calicified zone lies between the cartilage of the radial zone and in addition to the radially aligned collagen it contains a mineral component consisting of mainly ellipsoidal calcium hydroxyapatite crystals $\left[\mathrm{Ca}_{10}\left(\mathrm{PO}_{4}\right)_{6}(\mathrm{OH})_{2}\right]$ [2]. The boundary between the calcified and non-calcified cartilage is referred to as the tidemark and the interface between the calcified cartilage and the subchondral 
bone is called the cement line. Previous histochemical investigations have been seriously compromised by the tehcnical problems of preparting histological sections of this region.

The cells, chondrocytes, are responsible for the maintenance of the extracellular matrix, a process which is largely regulated by the communication of physical signals through the surrounding matrix [3]. The pericellular matrix is a thin zone, different in composition to the interterritorial matrix, which surrounds each cell [4] and is believed to be an important element in this comunication.

Here the challenge to microscopy has been that of preseving cell and matirx architecture during processing. Due, largely to these difficulties many important questions concerning the relationships between the structure of the matrix and its biomechanical properties are still unresolved. In consequence, the functional implications of biochemical changes, and even the sequence of cause and effect, occurring in osteoarthritis are still unclear.
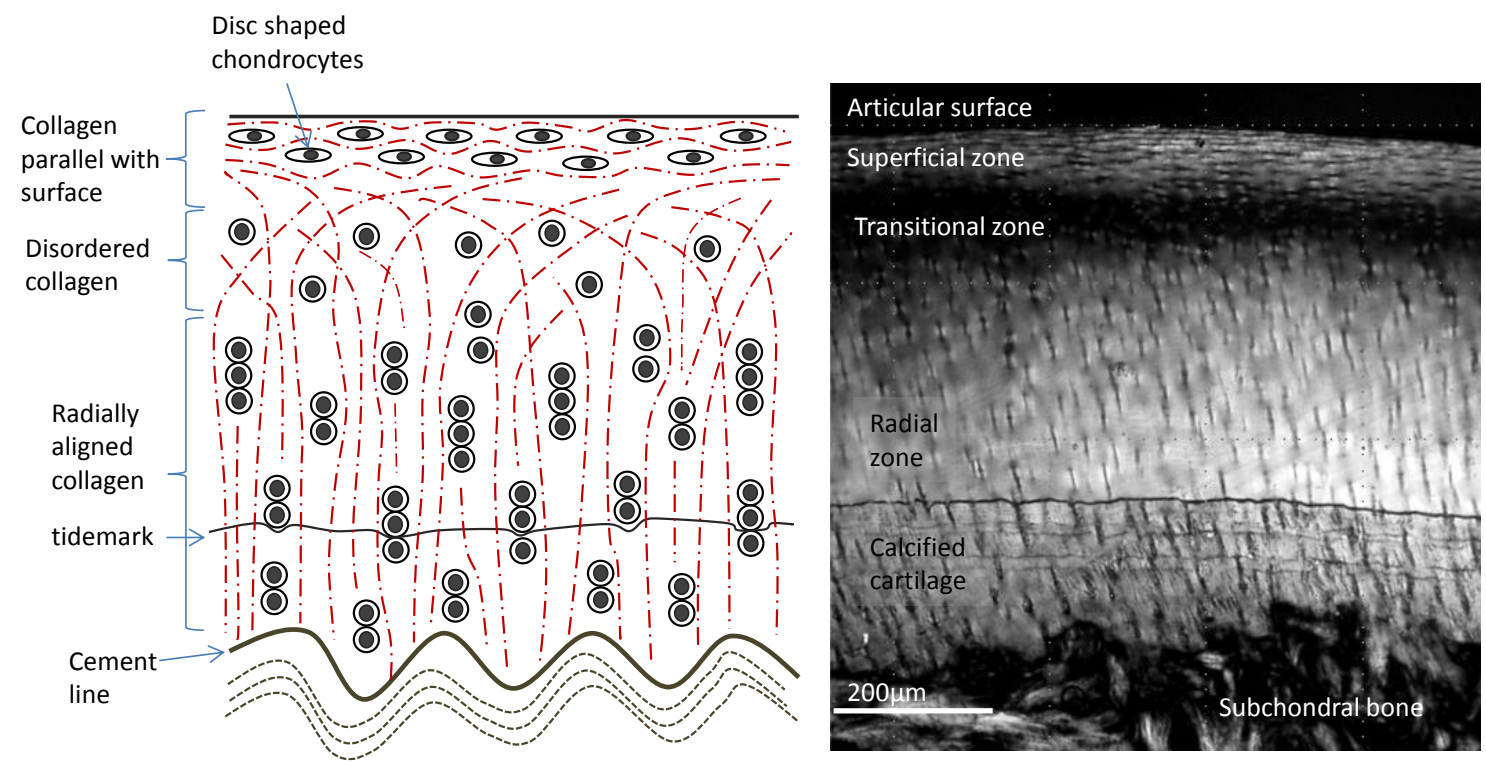

Figure 1, The zonal structure of articular cartilage. LHS schematic diagram of cartilage showing the collagen fibre orientations and the cells shapes as a function of depth. RHS $20 \mu \mathrm{m}$ thick histological section of cartilage viewed between crossed polarizers.

In the last few years there have been a number of applications of nonlinear microscopy to cartilage. Second harmonic (SHG) imaging of collagen is well established $[5,6]$. Its application to cartilage is complicated by the fact that the individual fibres of type II collagen are finer than those of type I and below the limit of resolution, but valuable information on its organisation in cartilage has been derived by image processing $[7,8]$ and polarization senstive measurements [9]. Elastin shows strong two photon fluorescence (TPF) and this technique has revealed the somewhat unexpected presence of an extensive network of elastin fibres in the surface zone of articular cartilage [10,11]. Whilst SHG and TPF are extremely useful in exploring the architecture of fibrous protein matrices there is a need for more general approaches capable of imaging the distribution of other tissue constituents.

In order to obtain information on the chemical composition of tissue without using histological staining techniques, Raman microscopy techniques which gain contrast from the vibrational modes of chemical bonds can be used. Some success has been reported in the application of spontaneous 
Raman imaging to cartilage [12]. However, there are technical limitations because Raman imaging is extremely slow due to the small Raman scattering cross section and penetration and spatial resolution, particularly in depth, are limited so most data have been obtained on histological sections. Coherent Raman overcomes many of these problems due to an increased interaction crosssection and intrinsical confocalilty allowing $3 \mathrm{~d}$ submicron resolution imaging. The most widely used method, Coherent anti-Stokes Raman Scattering (CARS), was first demonstrated in 1999 [13] and has been applied to a number of biological systems[14], including cartilage [11], with promising results. CARS provides contrast for the lipids in cells and tissues and combined with complimentry infromation from SHG and TPF imaging is a powerful technique for investigating tissue structure. However, the technique suffers from a high non-resonant background and an associated loss of spectral resolution. This means that often the chemically specific contrast in CARS is only allows imaging of lipid rich structures and does not make use of the wealth of chemical infromation that can be obtained in spontaneous Raman.

Stimulated Raman Scattering (SRS) has recently emerged as an alternative to CARS and has two distinct advantages for chemical specific imaging; firstly there is no complication due to a nonresonant background, meaning that the peaks in SRS spectra correspond directly with those in spontaneous Raman, and secondly the SRS signal is linearly dependent on the number of scatters compared to a quadratic dependence in CARS simplifying quantitative analysis of image data[15-18].

The aim of the present work was to explore the potential of both approaches in providing biologically relevant information on cartilage structure. We demonstrate that SRS provides straight forward in-situ chemical analysis on the subcellular scale with the same degree of specificity available with spontaneous Raman. The spatial resolution of the technique enabled us to explore the composition of the pericellular matrix and variations in matrix properties throughout the calcified zone

\section{Experimental}

\subsection{Materials}

Fresh equine metacarpophalangeal joints were collected from a local abattoir (Potters, Taunton, UK) and dissected in the laboratory. Cartilage samples were taken from specimens showing no macroscopic degeneration. For imaging cells in and the pericellular martix samples were prepared by removing slices of articular cartilage from the joint with a scapel blade and mounting them between a two coverslips with a film of physiological saline so that the imaging plane was parallel with the articular surface. For investigating the mineralized tissue thick saggital sections were cut from the distal end of the proximal phalanx using a freezing microtome.

\subsection{Multi-modal Microscopy}

Imaging was carried out on a custom-built multi-modal microscope comprising of a modified inverted microscope and confocal laser scanner (IX71 and FV300, Olympus UK). To optimise the transmission of the near IR light needed for CARS and SRS imaging, the standard galvanometer scanning mirrors were replaced with silver galvanometric mirrors and the tube lens was replaced by a MgF2 coated lens. The dichroic mirror within the scan unit was replaced by a silver mirror which 
gave high reflectivity throughout the visible and NIR (21010 Chroma Technologies). The light was focused onto the sample using a 60x 1.2NA water immersion objective (UPlanS Apo, Olympus UK)

\subsubsection{SHG and TPF:}

Both SHG and TPF were excited using the 800nm output from a mode-locked femto-second Ti:Sapphire laser (Mira $900 \mathrm{D}$ Coherent) with a pulse width of approximately $100 \mathrm{fs}$ and a $76 \mathrm{MHz}$ repetition rate. The signal was collected in the epi-direction using the objective lens and separated from the laser fundamental using a long pass dichroic mirror (670dcxr Chroma Technologies). The light was focused on to a PMT (R3896, Hamamatsu). The following filter combinations (CG-BG-39 and F10-400-5-QBL, CVI laser) and (CG-BG-39 and F70-500-3-PFU, CVI laser) were used to isolate the SHG and TPF light respectively.

\subsubsection{SRS:}

For Stimulated Raman Scattering (SRS) the Stokes beam was provided by the $1064 \mathrm{~nm}$ pico-second output of a Nd:Vandium pico-second laser (High-Q Laser Production $\mathrm{GmbH}$ ). To produce the pump beam the frequency doubled output of the $\mathrm{Nd}$ :Vandium laser was used to pump an optical parametric oscillator (OPO) (Levante Emerald, APE, Berlin, Germany). The idler beam from the OPO was blocked and the signal beam was used as a pump beam. Both the pump and Stokes beams consisted of $6 \mathrm{ps}$ pulses with a $76 \mathrm{MHz}$ repetition rate. The signal beam from the OPO was continuously tuneable between 690 and 990nm and therefore allowed Raman shifts between 5092 and $702 \mathrm{~cm}^{-1}$ to be investigated.

The Stokes beam was modulated at a high frequency $1.7 \mathrm{MHz}$ using an electro-optic modulated Pockels cell (Leysop EM200). The EOM produced modulations in the polarization state of the beam and these were converted into modulations in amplitude at a Glan-Taylor polarizing beam splitter. To enhance the sharpness of the intensity modulations a quarter and a half wave-plate were also included in the beam path. An optical delay stage was included in the Stokes beam path before it was combined with the pump beam to allow the two beams to be overlapped in time. The pump and Stokes beams were combined at a short pass dichroic mirror.

The SRS process within the sample was detected by measuring the Stimulated Raman Loss (SRL) in the pump beam in the epi-direction. Transmitted light was collected using a 60x 1.0NA dipping lens (Fluor X60, Nikon, Japan). The pump beam was spectrally isolated from the Stokes beam by a band pass filter (CARS 890/220nm, Chroma technologies) and detected using a wide area Si photo-diode (FDS1010 Thorlabs). To suppress the strong signal due to the laser pulsing at $76 \mathrm{MHz}$, the output current was filtered by a low pass filter (mini-circuits, BLP-1.9+) and then terminated by a $50 \Omega$ resistor. The photo-diode was connected to a radio frequency lock-in amplifier (Stanford Research, SR 844RF) which detected the modulations in the pump beam at the $1.7 \mathrm{MHz}$ frequency set by the EOM. This allowed the low frequency fluctuations due to laser noise to be excluded. The modulus of the output from the lock-in amplifier $\left(\Delta I_{p}\right)$ was fed into the microscope analogue to digital converter. A pixel dwell time of $100 \mu$ s was required for SRS imaging as this corresponds to the fastest integration time of the lock-in amplifier.

SRS spectra were obtained by tuning the wavelength of pump beam. Images were taken at $0.2 \mathrm{~nm}$ intervals of the pump beams wavelength. In order to compensate for any changes in the intensity of the pump beam caused by the tuning the laser the overall intensity of the pump beam $I_{p}$ measured 
at the photo-diode was recorded in a second channel. To produce a value of $\Delta I_{p} / I_{p}$ the output of the modulated channel was divided by the overall output.

\subsubsection{CARS:}

CARS imaging could be performed simultaneously with the SRS imaging using the same laser set-up. The anti-stokes beam was collected on to a PMT (R3896, Hamamatsu) in the epi-direction. To separate it from the laser fundamental the anti-Stokes beam was reflected off a long pass dichroic (750dcxr Chroma) and passed through 2 band pass filters centred at 660nm (Ealing 660.0 IF 40-D)

\subsubsection{Raman Micro-Spectroscopy:}

Raman spectra were acquired on a Renishaw RM1000 Raman Microscope (Renishaw plc, UK). This microscope was set up to collect spectra in the back-scattered direction. The output from a $785 \mathrm{~nm}$ HeNe continuous wave laser with a maximum power at the sample of $300 \mathrm{~mW}$ was used. A spectral grating with 1200 lines $/ \mathrm{mm}$ was used, which gave a spectral resolution of $1 \mathrm{~cm}^{-1}$. The spectra were collected using a 40x 0.55NA microscope objective (Leica Germany). The samples were mounted on aluminised slides and covered with a quartz coverslip, to avoid dehydration of the sample during data collection. The reported spectra were calculated by averaging 5 spectra and the fluorescent background was removed using the baseline subtraction function in wire.

\subsubsection{Peak fitting:}

Gaussian-lorentzian peaks were fitted to the $\mathrm{CH}$ region of the spectra in matlab using the curve fit toolkit (Matlab R2012a). For initial peak positions and boundry conditions peaks identified in the literature were used.

\section{Results and discussion}

In section 3.1 we introduce spontaneous Raman spectra for unmineralised cartilage, calcified cartilage and bone. We identify peaks at $959 \mathrm{~cm}^{-1}$ and $1070 \mathrm{~cm}^{-1}$ for the imaging of mineral. In section 3.2 we demonstrate that SRS gives more spectral information for imaging in thick cartilage samples compared to CARS. In section 3.3 we investigate the SRS spectra in more detail, comparing SRS spectra from different structures within the images and comparing these to spontaneous Raman taken on purified tissue components. In sections 3.4 and 3.5 SRS is used to image chondrocytes and their pericellular environment and mineralized tissue.

\subsection{Preliminary Raman Investigation}

In order to identify possible Raman resonances for SRS imaging, spontaneous Raman spectra were obtained for cartilage, calcified cartilage and the subchondral bone. These are shown in figure 2 and the peak assignments are given in table 1 . The Raman spectrum of un-calcified cartilage is mostly dominated by peaks from the type II collagen. However a clear marker of the proteoglycan content is a peak at around $1058-1064 \mathrm{~cm}^{-1}$ which corresponds to the $\mathrm{OSO}_{3}{ }^{-}$symmetric stretch [19]. This peak disappears following guanidine extraction of the proteoglycans from the sample (data not shown). In both bone and calcified cartilage there is a very strong peak at $959 \mathrm{~cm}^{-1}$ due to phosphate $\left(\mathrm{PO}_{4}{ }^{3-}\right)$ 
vibrations in the hydroxyapaptite. In addition, there is a weaker peak at $1070 \mathrm{~cm}^{-1}$ due to carbonate $\left(\mathrm{CO}_{3}{ }^{2-}\right)$ vibrations where some of the phosphate groups have been substituted for carbonate[2].

The $\mathrm{CH}$ vibrational region between 2800 and $3000 \mathrm{~cm}^{-1}$ is of particular interest to CARS and SRS studies as these Raman active vibrations produce strong imaging contrast. Spectroscopic studies across this region provide information on the chemical compositions of the samples due to differences in the environment of the $\mathrm{CH}$ bonds. For aliphatic molecules there are two predominant peaks at 2840 and $2870 \mathrm{~cm}^{-1}$ corresponding to the $\mathrm{CH}_{2}$ symmetric and anti-symmetric stretch, whereas proteins are characterised by a peak at $2930 \mathrm{~cm}^{-1}$ and a shoulder at $2870 \mathrm{~cm}^{-1}[20,21]$. 
Table 1 Raman peaks found in cartilage, calcified cartilage and bone[2, 19, 22, 23]

\begin{tabular}{|c|c|c|c|c|c|}
\hline $\begin{array}{l}\text { Raman } \\
\text { shift } \mathrm{cm}^{-1}\end{array}$ & Description & Assignment & $\begin{array}{l}\text { cartilag } \\
\text { e }\end{array}$ & $\begin{array}{l}\text { Calcified } \\
\text { cartilage }\end{array}$ & Bone \\
\hline 2970 & shoulder & $\mathrm{CH}_{3}$ asymmetric & $\checkmark$ & $\checkmark$ & $\checkmark$ \\
\hline 2930 & strong & $\mathrm{CH}_{3}$ symmetric & $\checkmark$ & $\checkmark$ & $\checkmark$ \\
\hline 2870 & shoulder & $\mathrm{CH}_{2}$ asymmetric & $\checkmark$ & $\checkmark$ & $\checkmark$ \\
\hline 2840 & weak & $\mathrm{CH}_{2}$ symmetric & $\checkmark$ & $\checkmark$ & $\checkmark$ \\
\hline 1664 & $\begin{array}{l}\text { Narrow, } \\
\text { strong }\end{array}$ & Amide I & $\checkmark$ & $\checkmark$ & $\checkmark$ \\
\hline 1651 & shoulder & Amide I & $\checkmark$ & $\checkmark$ & $\checkmark$ \\
\hline 1607 & Shoulder & Phe, Thr & $\checkmark$ & $\checkmark$ & $\checkmark$ \\
\hline 1554 & weak & tryptophan & $\checkmark$ & $\checkmark$ & $\checkmark$ \\
\hline 1451 & $\begin{array}{l}\text { Narrow, } \\
\text { strong }\end{array}$ & $\mathrm{CH}_{2}$ and $\mathrm{CH}_{3}$ & $\checkmark$ & $\checkmark$ & $\checkmark$ \\
\hline 1426 & $\begin{array}{l}\text { Narrow, } \\
\text { weak }\end{array}$ & $\mathrm{CH}_{2}$ & $\checkmark$ & $\checkmark$ & $\checkmark$ \\
\hline 1379 & $\begin{array}{l}\text { Narrow, } \\
\text { weak }\end{array}$ & & $\checkmark$ & $\checkmark$ & $\checkmark$ \\
\hline 1340 & medium & $\mathrm{CH}_{2}$ and $\mathrm{CH}_{3}$ & $\checkmark$ & $\checkmark$ & $\checkmark$ \\
\hline 1319 & Medium & $\mathrm{CH}_{2}$ & $\checkmark$ & $\checkmark$ & $\checkmark$ \\
\hline 1270 & shoulder & Amide III & $\checkmark$ & $\checkmark$ & $\checkmark$ \\
\hline 1245 & $\begin{array}{l}\text { Narrow, } \\
\text { strong }\end{array}$ & Amide III & $\checkmark$ & $\checkmark$ & $\checkmark$ \\
\hline 1125 & weak & C-N stretching & $\checkmark$ & $\checkmark$ & $\checkmark$ \\
\hline 1070 & $\begin{array}{l}\text { Narrow, } \\
\text { strong }\end{array}$ & $\mathrm{CO}_{3}{ }^{2-}$ & & $\checkmark$ & $\checkmark$ \\
\hline 1058-64 & $\begin{array}{l}\text { Narrow, } \\
\text { strong }\end{array}$ & $\begin{array}{l}\mathrm{OSO}_{3}^{-} \\
\text {Proteoglycans }\end{array}$ & $\checkmark$ & & \\
\hline 1031 & Narrow & Phenylalanine & $\checkmark$ & $\checkmark$ & $\checkmark$ \\
\hline 1002 & $\begin{array}{l}\text { Narrow, } \\
\text { strong }\end{array}$ & Phenylalanine & $\checkmark$ & $\checkmark$ & $\checkmark$ \\
\hline 959 & $\begin{array}{l}\text { Narrow, } \\
\text { very, strong }\end{array}$ & $\mathrm{PO}_{4}^{3-}$ & & $\checkmark$ & $\checkmark$ \\
\hline 937 & $\begin{array}{l}\text { Narrow, } \\
\text { strong }\end{array}$ & $\mathrm{C}-\mathrm{O}-\mathrm{C}$ & $\checkmark$ & & \\
\hline 877 & shoulder & C-C stretching & $\checkmark$ & $\checkmark$ & $\checkmark$ \\
\hline 855 & & $\mathrm{C}-\mathrm{C}-\mathrm{H}$ & $\checkmark$ & $\checkmark$ & $\checkmark$ \\
\hline 816 & & C-O-C stretching & $\checkmark$ & $\checkmark$ & $\checkmark$ \\
\hline
\end{tabular}




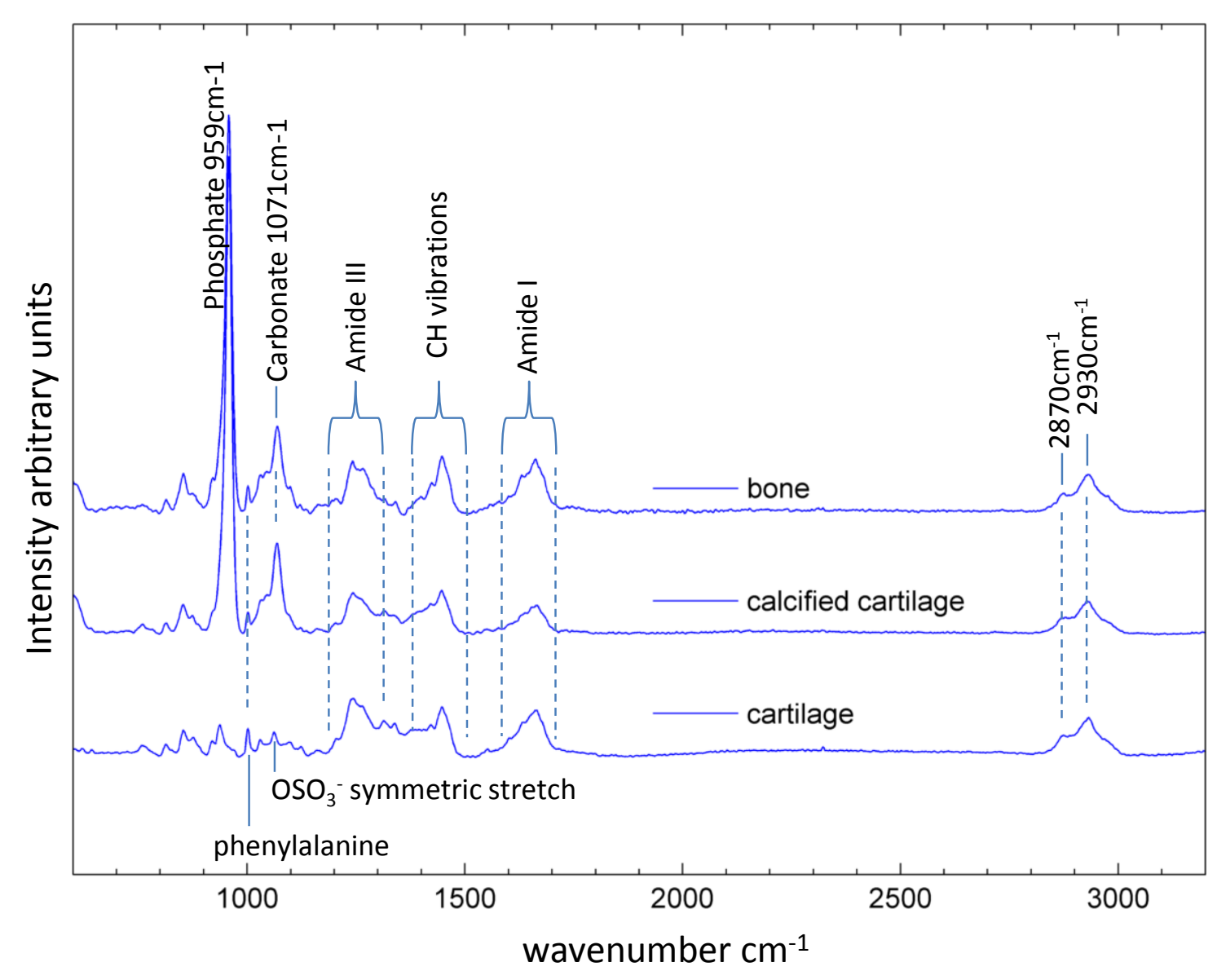

Figure 2. The spontaneous Raman spectra of cartilage, calcified cartilage and bone.

\subsection{Comparison of CARS and SRS}

Cartilage was imaged simultaneously with CARS and SRS tuned to the $\mathrm{CH}$ vibrational region between $2800-3000 \mathrm{~cm}^{-1}$. In both modalities the cells and the extra-cellular matrix can be clearly visualised (see figure 3B and C) however the spectra are clearly different (see figure 3A). In the CARS imaging there is a large contribution from non-resonant four wave mixing which corresponds to approximately $50 \%$ of the overall intensity even on resonance (see figure $3 \mathrm{~A}$ ). The non-resonant signal does not contain any chemically specific information and contrast is based on differences in polarizability and refractive index [24]. The spectra shape of the spectra is also complicated by the mixing of the resonant and non-resonant contributions to the signal resulting in a dispersive peak shape with dip in the spectra slightly blue shifted from the main peak caused by distructive interference between the resonant and non-resonant terms [14]. In the SRS spectra the signal almost disappears off resonance and therefore allowing chemically specific imaging. The small background in the SRS images may be due to a combination of photo-thermal effects $[25,26]$ and two-photon two-colour absorption [27, 28] or cross phase modulation. 

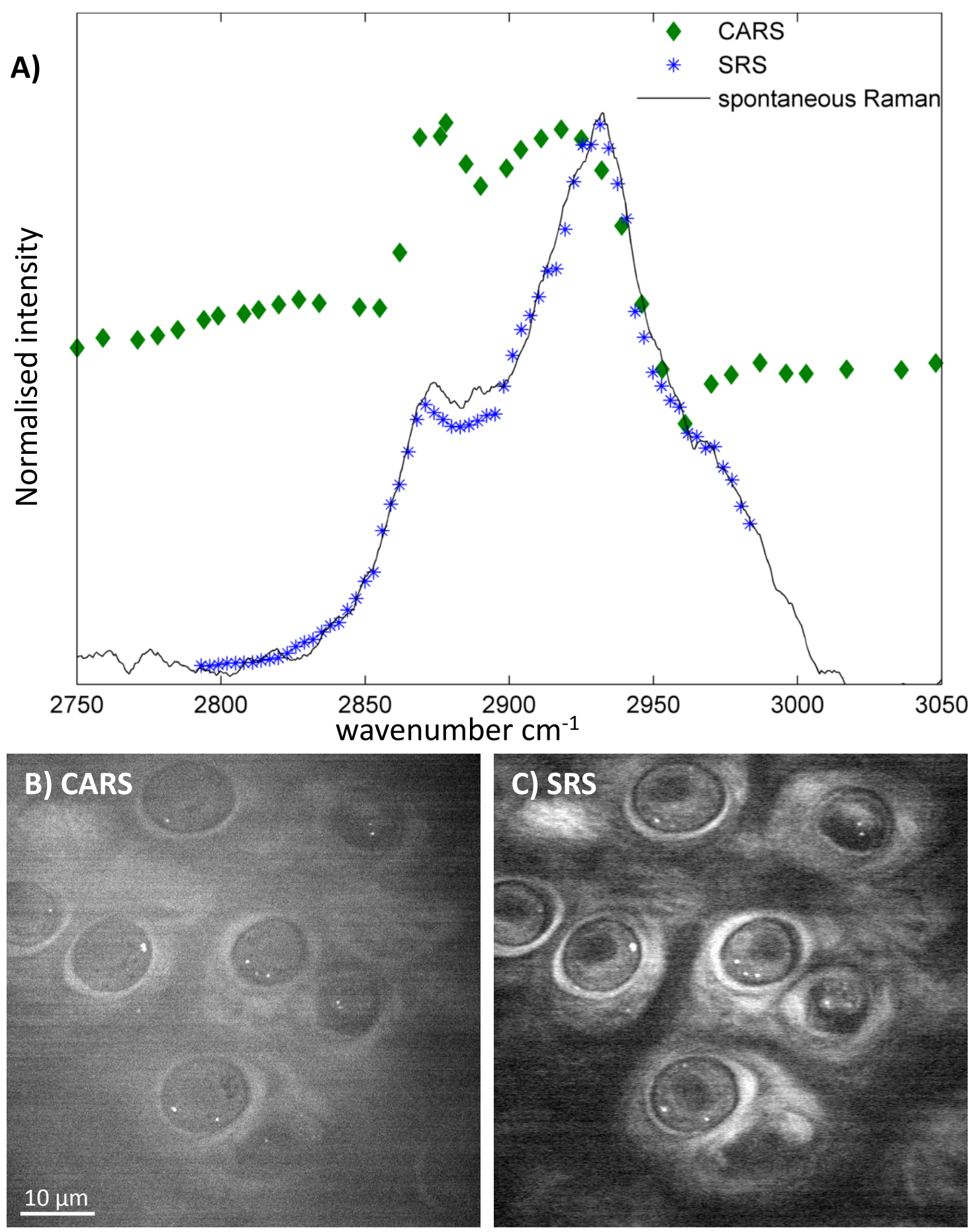

Figure 3. CARS and SRS in cartilage. A) CARS, SRS and spontaneous Raman spectra of the cartilage extra-cellular matrix taken across the $\mathrm{CH}$ region demonstrating the high non-resonant background in the CARS images. B) CARS image of superficial zone chondrocytes taken at $2840 \mathrm{~cm}^{-1}$ and C) SRS image of the same area taken at $2840 \mathrm{~cm}^{-1}$

\subsection{Coherent Raman Spectroscopy in the $\mathrm{CH}$ region}

The SRS spectrum in the range $2800-3000 \mathrm{~cm}^{-1}$ of the extracellular matrix is shown in figure 4B, along with the spontaneous Raman spectra of cartilage, collagen type II, and aggrecan. The SRS from the extra-cellular matrix shows excellent correlation with the Raman spectra of bulk cartilage matrix, type II collagen. All three spectra show a strong peak at $2930 \mathrm{~cm}^{-1}$ and another peak of about half the intensity at $2870 \mathrm{~cm}^{-1}$ and a shoulder at around $2968 \mathrm{~cm}^{-1}$. The spectrum of aggrecan differs from the 
other spectra and with a strong peak at $2930 \mathrm{~cm}^{-1}$ and shoulders at around 2906 and $2970 \mathrm{~cm}^{-1}$. The extra-cellular matrix spectrum is dominated by the collagen content and not the proteoglycan content.

In the pericellular matrix of the superficial zone chondrocytes there was an additional peak at $2840 \mathrm{~cm}^{-1}$ as shown in figure $4 \mathrm{C}$. This indicates the presence of lipid within the pericellular matrix. Lipid droplets have been observed in the pericellular matrix in electron microscopy studies [29] however the droplet size would be beneath the resolution limit of our imaging technique with the largest being $0.2 \mu \mathrm{m}$. As in the extra-cellular matrix the pericellular matrix has a large peak at $2930 \mathrm{~cm}^{-1}$ indicating a high protein content.

The spectra of the chondrocyte and lipid droplets within it are shown in figure 4D along with the spontaneous Raman spectrum of isolated chondrocytes for comparison (The chondrocytes were extracted by enzyamtic digestion of the cartilage and supplied by kind gift of Dr.J.Urban Oxford). The Raman spectrum of isolated chondrocytes represents the average cell contents including the nucleus, organelles and cytoplasm whereas the SRS enabled us to investigate the spectra of the lipid droplets and the cytoplasm. The spectra of lipids depends strongly on the degree of saturation and the crystaline order within the sample. Saturated fatty acids have an ordered arrangement at room temperature and this results in a strong peaks for both the symmetric and antisymmetric $\mathrm{CH}_{2}$ stretches. The ratio of these peak heights is dependent on the crystallinity of the sample in crystaline samples the anti-symmetric peak is more intense than the symmetric peak [30]. Unsaturated fatty acids are liquid at room temperature, in their spectra the $2840 \mathrm{~cm}^{-1}$ peak is still present but the peak at $2870 \mathrm{~cm}^{-1}$ is less pronounced and incorporated in a much broader peak between arround 2860 $2930 \mathrm{~cm}^{-1}[31,32]$. The spectrum of the chondrocyte lipid droplets matches that of unsaturated fatty acids, this is in good aggrement with the data on the overall lipid content of cartilage reported in the literature with the most abundant fatty acids being oleic, palmitic and linoleic (listed in order of decreasing abundance) [33]. 

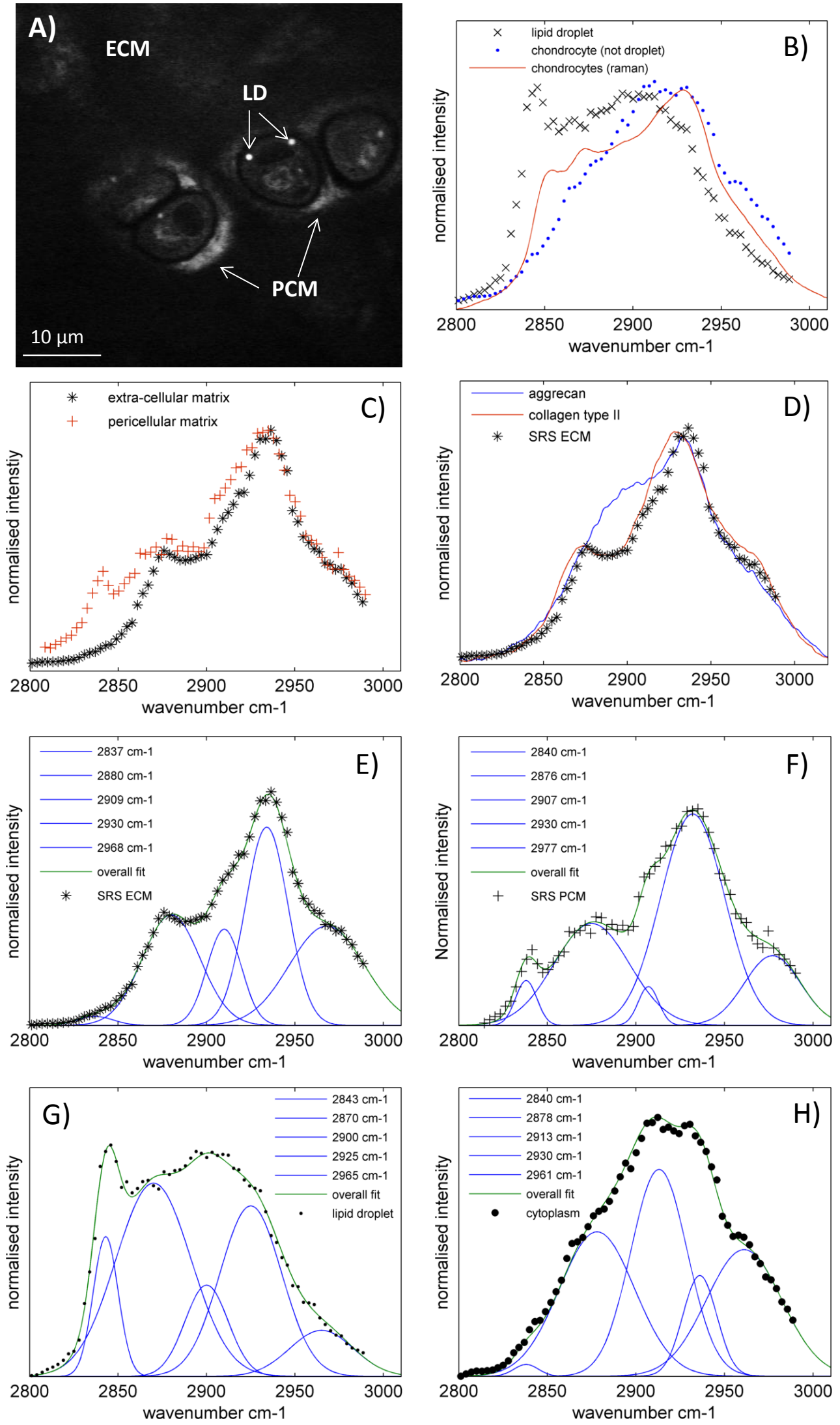

Figure 4. SRS spectroscopy of the $\mathrm{CH}$ region in cartilage. A) SRS image of cartilage indicating the different regions of interest. B) SRS spectrum of the ECM compared to spontaneous Raman of its two major components type II collagen and aggrecan C) SRS spectra of the pericellular matrix compared to the ECM. D) SRS spectra of lipid droplets and cytoplasm compared to spontaneous Raman of purified chondrocytes. E-H) Gaussian peaks fitted to the spectra of the ECM, PCM, lipid droplet and cytoplasm respectively. 


\subsection{Imaging of cells and matrix}

SRS images were taken of the cells and their pericellular environment at different Raman shifts within the $\mathrm{CH}$ vibrational region $\left(2840 \mathrm{~cm}^{-1}, 2870 \mathrm{~cm}^{-1}\right.$ and $\left.2930 \mathrm{~cm}^{-1}\right)$ alongside TPF and SHG images to provide additional information on the elastin and collagen networks (See figures 5 and 6). Colour merged images generated from the $2840 \mathrm{~cm}^{-1}$ peak (red) and the $2930 \mathrm{~cm}^{-1}$ peak (green) were also obtained to visualise the relative distributions of protein and lipid. Regions with a high protein content but low lipid content appear green on the images, regions with a high lipid and protein content appear orange or yellow and regions with a high lipid content appear red. Cells in both the superficial and deep zones were investigated.

The images taken at $2840 \mathrm{~cm}^{-1}$ gave a highest contrast between the nucleus and the surrounding cytoplasm due to its lower lipid content [34]. It has been reported that in some cases where nuclei contain many replica choromosones SRS can be used to image the nucleic acid content via the $785 \mathrm{~cm}^{-1}$ vibration for pyrimidine bases [34]. Imaging at this raman shift was attempted in the cartilage however we found there was insufficient contrast to use this Raman shift to image the nucleus. In the superficial zone the pericellular matrix showed up brightly at $2840 \mathrm{~cm}^{-1}$ (figure 5A) and also in the TPF image (figure $5 \mathrm{E}$ ). Both the increased pericellular fluorescence and lipid signal were absent from the pericellular matrix of cells in the deep zone (figure $6 \mathrm{~A}$ and $\mathrm{E}$ ). However lipid droplets were observed within the cells in both zones.

In the images taken at $2930 \mathrm{~cm}^{-1}$ both the cells, periclellular matrix and extracellular matrix appear bright. The contrast in the extracellular matrix arises from the type II collagen which has also been imaged with SHG (figures $5 \mathrm{~F}$ and $6 \mathrm{~F}$ ). The amidel peak around $1660 \mathrm{~cm}^{-1}$ also investigated as a source of contrast for protein, wavelength dependent SRS at this Raman shift was detectable but the contrast was much less and therefore this peak was not used in the imaging. When the SRS images are compared to the SHG images of the collagen matrix the fibrous nature of the collagen matrix is more apparent in the SHG images which contain coarser textural features. The observation holds true in both the deep and superficial zones. A similar observation was reported by Pfeffer et al when comparing CARS and SHG images of type I collagen in tendon [35]. This may be because the SRS signal contains contributions from additional $\mathrm{CH}$ bonds which are not within the collagen fibres, for example the $\mathrm{CH}$ bonds within the proteoglycans. Alternatively it is possible that this may be because the SHG is dependent on the square of the number of scatterers while the SRS is linearly dependent on the number of scatterers. In addition the SHG from collagen is dependent on the distribution, order and orientation of the collagen molecules molecules[36-38]. 


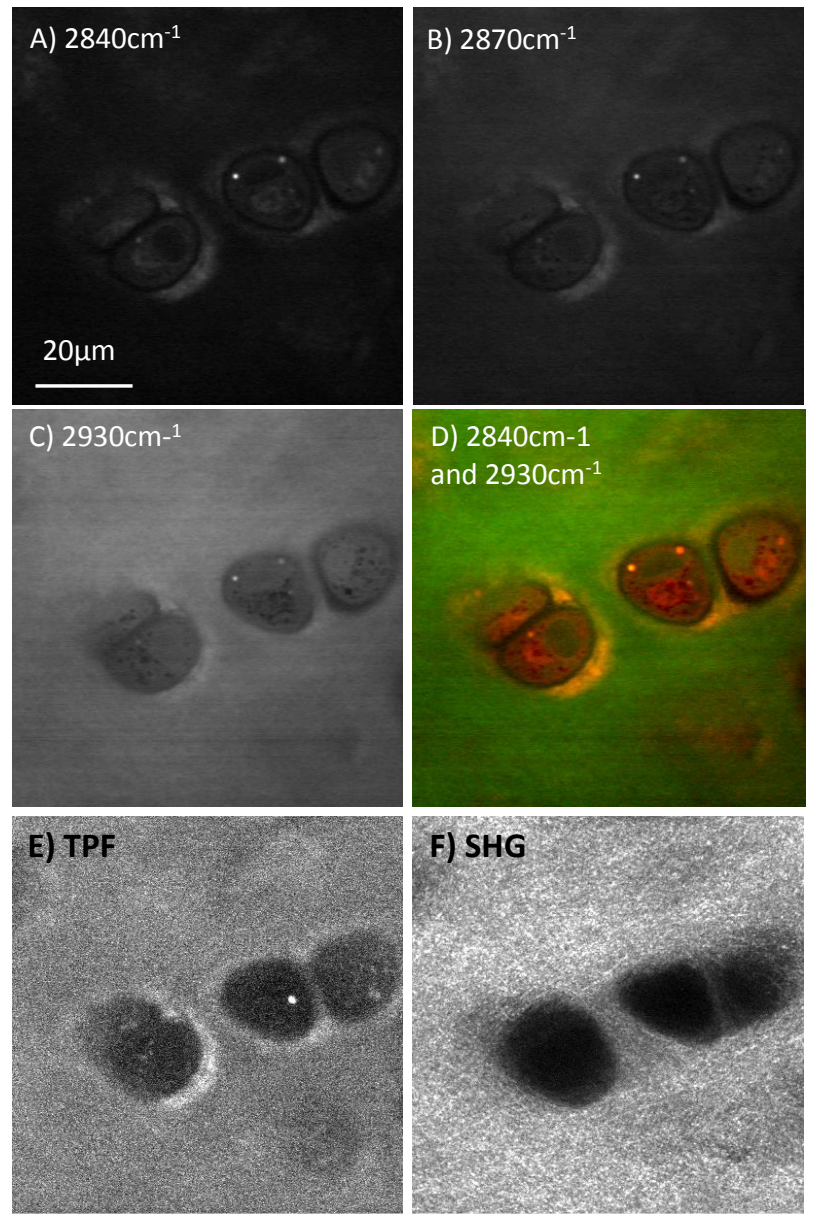

Figure 5 Chondrocytes from the superficial zone. (A-D) SRS images taken at different Raman shifts to highlight lipid and protein content. D) is the result of merging images the image at $2840 \mathrm{~cm}^{-1}$ (red) with the image at $2930 \mathrm{~cm}^{-1}$ (green) to show relative distributions of protein and lipid. E) TPF image showing autofluorescence centred at 500nm and F) SHG image showing the network of collagen II fibres 

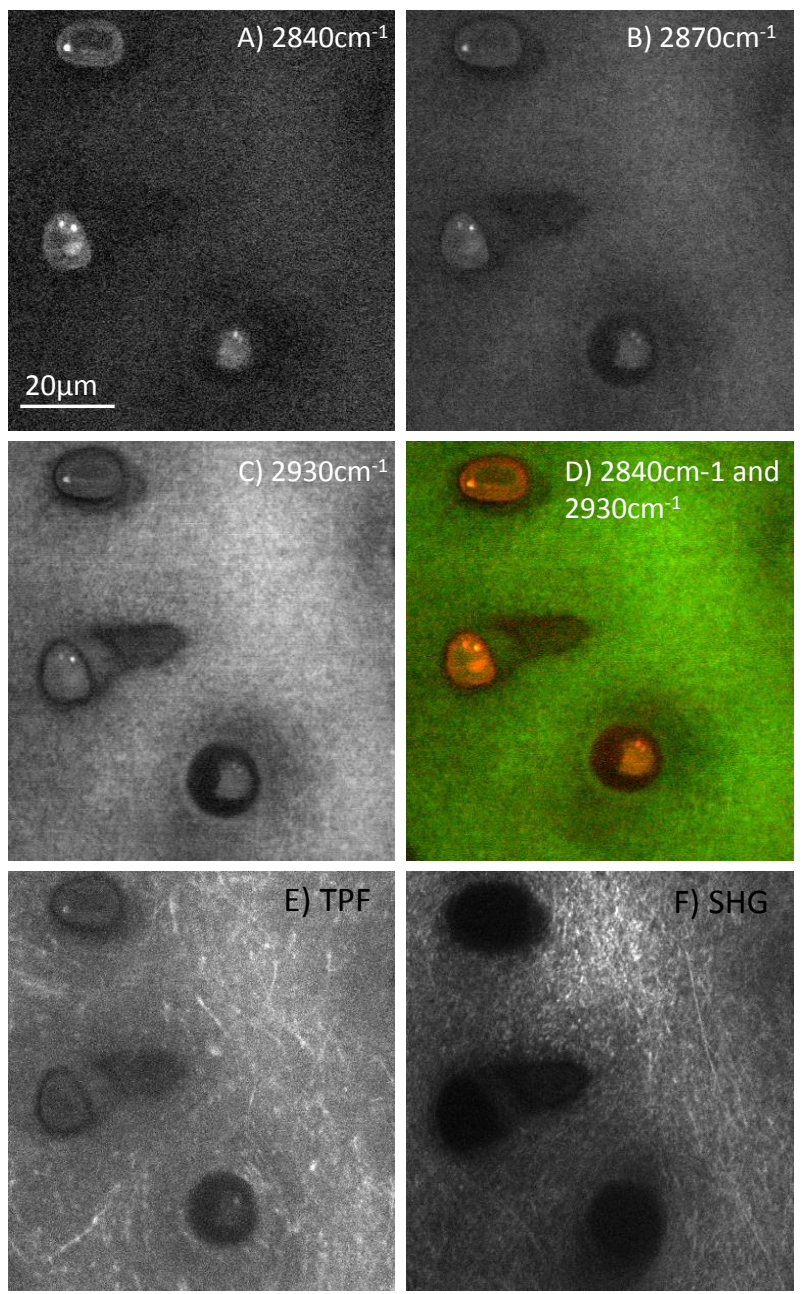

Figure 6 Chondrocytes from the radial zone. (A-D) SRS images taken at different Raman shifts to highlight lipid and protein content. D) is the result of merging images the image at $2840 \mathrm{~cm}^{-1}$ (red) with the image at $2930 \mathrm{~cm}^{-1}$ (green) to show relative distributions of protein and lipid. E) TPF image showing autofluorescence centred at 500nm and F) SHG image showing the network of collagen II fibres

\subsection{SRS imaging of calcified tissue:}

The SRS spectrum of the mineralized tissue showed both the phosophate and carbonate peaks indentified in the spontaneous Raman spectra (see figure 7). Both peaks were absent in the nonmineralized tissue. 


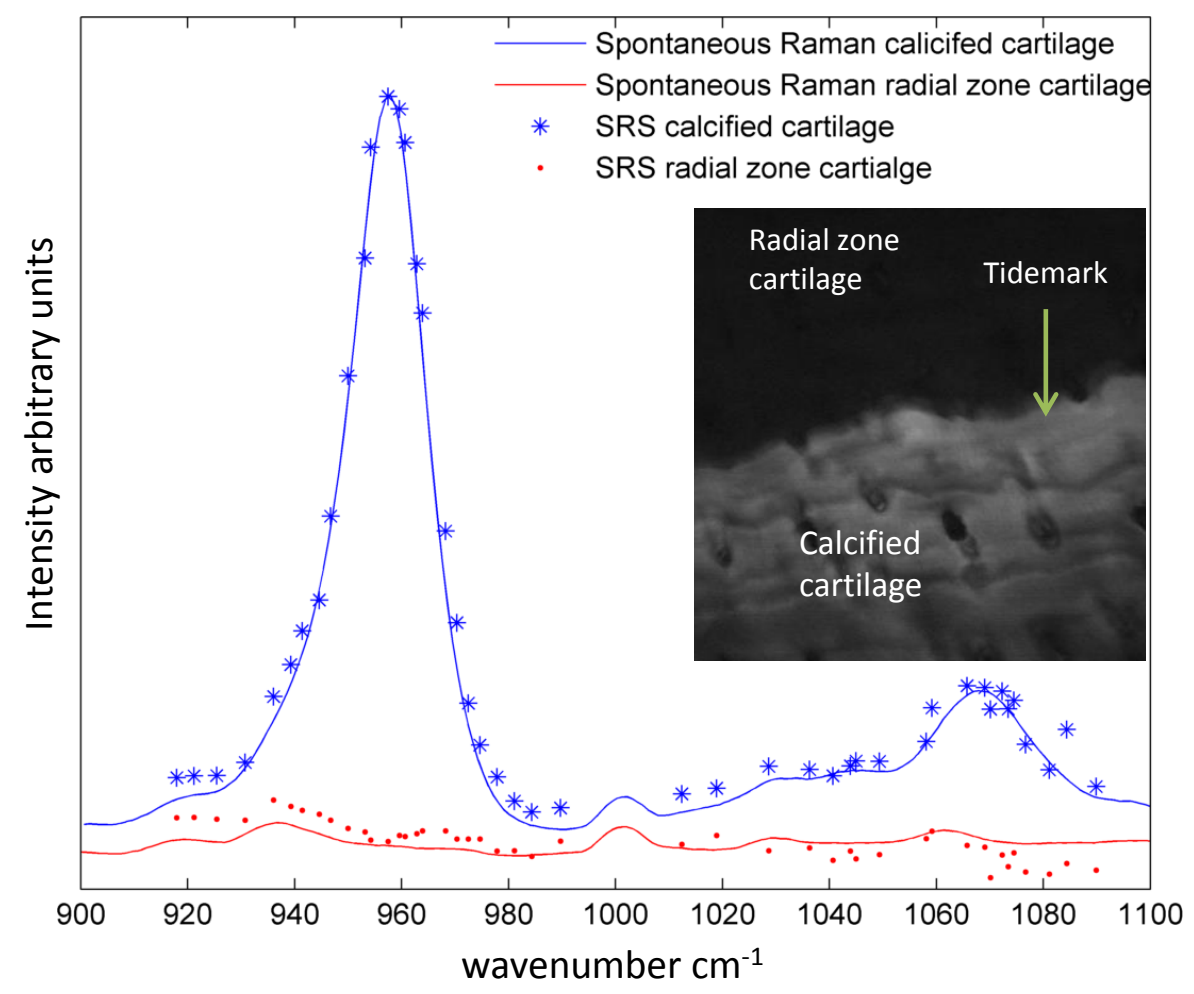

Figure 7 SRS and spontaneous Raman spectra taken in calcified cartilage showing the phosphate peak at $959 \mathrm{~m}^{-1}$ and the carbonate peak at around $1070 \mathrm{~cm}^{-1}$. SRS spectra of the radial zone cartilage from the same area is also included to demonstrate these peaks are absent from the non-calcified tissue. (inset shows area of cartilage from which the spectral data was taken)

Large area images were taken of the bone-cartilage interface to include both the tidemark, zone of calcified cartilage and the underlying subchondral bone. For this a combination of imaging modalities was applied, SRS at $2840 \mathrm{~cm}^{-1}$ for lipids, $1070 \mathrm{~cm}^{-1}$ for carbonate and $959 \mathrm{~cm}^{-1}$ for phosphate, along with TPF and SHG. Although the phosphate peak is stronger than the carbonate peak and therefore offers better image contrast it was important to image at both wavenumbers as differences in the ratio of carbonate to phosphate can be related to tissue formation and maturation [39]. Both the carbonate and phosphate images showed a step change in SRS signal across the tidemark reflecting the rapid change in mineral content. Lacunae and duplicate tidemarks were visible in all the imaging modalities. There was no appreciable change in mineral content between the bone and calcified cartilage. In the SRS images of the phosphate and carbonate peaks the cement line is not clearly defined, however it is clear in the SHG images (due to a change from type II collagen in the cartilage to type I collagen in the bone) and in the TPF images due to an increase in auto-fluorescence. Within the subchondral bone channels are clearly evident, and the SRS images taken at $2840 \mathrm{~cm}^{-1}$ show some large lipid stores within the channels. 

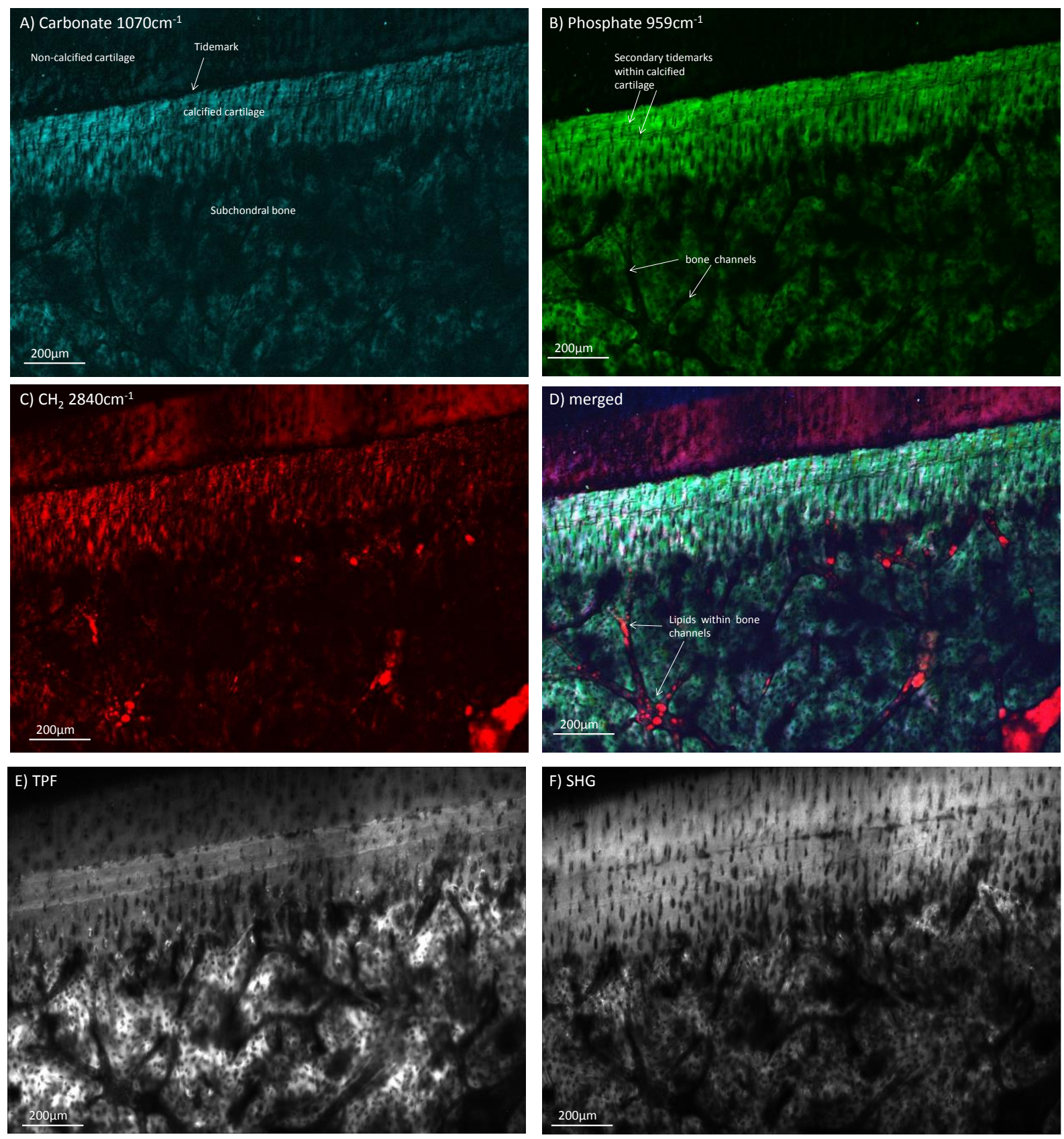

Figure 8. Large area images of the zone of calcified cartilage and subchondral bone. A-D) SRS imaging of carbonate $1070 \mathrm{~cm}$ ${ }_{1}^{1}(A)$, phsosphate $959 \mathrm{~cm}^{-1}(B)$ and lipids $2840 \mathrm{~cm}^{-1}(C)$. D) is a composite of A-C where $1070 \mathrm{~cm}^{-1}$ is blue, $959 \mathrm{~cm}^{-1}$ is green and $2840 \mathrm{~cm}^{-1}$ is red. E) and F) are TPF and SHG images of the same area respectively.

\section{Conclusion}

Spontaneous Raman provides a wealth of information on the composition of cartilage however due to slow acquisition times and poor penetration depths it is challenging to apply this technique to intact tissue samples. Coherent Raman imaging allows faster imaging times, and due to the infrared excitation wavelengths and intrinsic confocality it is much more suitable for imaging thick tissue samples. We have shown that the same chemical specificity as spontaneous Raman can be extracted using SRS with near-real-time data acquisition, 3D sectioning and submicron resolution. 
Hyperspectral SRS measurements provided information on the compostion of the extra-cellular matrix, peri-cellular matrix, chondrocyte cytoplasm and lipid droplets. This showed that there was lipid present in the pericellular matrix of superficial zone chondrocytes but not in the extra-cellular matrix. It also indicated that the lipid droplets in the chondrocytes contained unsaturated fatty acids. Imaging revealed differences between the superficial zone and the deep zone, most notably the pericellular lipid was only detectable in the superficial zone. SRS also allowed us to image the mineral content of cartilage.

In summary coherent Raman has allowed for the first time high resolution chemically specific imaging of both the cells and the mineral. Both the cells and the mineralized zone in the cartilage are key to understanding the progression of osteoarthritis and therefore coherent Raman provides a useful tool for future research.

\section{References}

[1] R.A. Stockwell, Biology of Cartilage Cells, Cambridge University Press, 1979.

[2] G. Penel, G. Leroy, C. Rey, E. Bres, Calcified tissue international, 63 (1998) 475-481.

[3] A.J. Grodzinsky, M.E. Levenston, M. Jin, E.H. Frank, Annual Review of Biomedical Engineering, 2 (2000) 691-713.

[4] C.A. Poole, Journal of Anatomy, 191 (1997) 1-13.

[5] S. Roth, I. Freund, Journal of Chemical Physics, 70 (1978) 1637-1643.

[6] P.J. Campagnola, A.C. Millard, M. Terasaki, P.E. Hoppe, C.J. Malone, W. Mohler, Biophys. J., 81 (2002) 493-508.

[7] J.C. Mansfield, C.P. Winlove, Journal of Anatomy, 220 (2012) 405-416.

[8] E. Werkmeister, N. de Isla, L. Marchal, J.F. Stoltz, D. Dumas, Biorheology, 45 (2008) 375-383.

[9] J.C. Mansfield, C.P. Winlove, J. Moger, S.J. Matcher, J. Biomed. Opt., 13 (2008) 044020.

[10] A.T. Yeh, M.J. Hammer-Wilson, D.C. Van Sickle, H.P. Benton, A. Zoumi, B.J. Tromberg, G.M. Peavy, Osteoarthritis Cartilage, 13 (2005) 345-352.

[11] J.C. Mansfield, J. Yu, D.P. Attenburrow, J. Moger, U. Tirlapur, J.P.G. Urban, Z.F. Cui, C.P. Winlove, Journal of Anatomy, 215 (2009) 682-691.

[12] A. Bonifacio, C. Beleites, F. Vittur, E. Marsich, S. Semeraro, S. Paoletti, V. Sergo, Analyst, 135 (2010) 3193-3204.

[13] A. Zumbusch, G.R. Holtom, X.S. Xie, Physical Review Letters, 82 (1999) 4142-4145.

[14] C.L. Evans, X.S. Xie, Annual Review of Analytical Chemistry, 1 (2008) 883-909.

[15] C.W. Freudiger, W. Min, B.G. Saar, S. Lu, G.R. Holtom, C.W. He, J.C. Tsai, J.X. Kang, X.S. Xie, Science, 322 (2008) $1857-$ 1861.

[16] P. Nandakumar, A. Kovalev, A. Volkmer, New Journal of Physics, 11 (2009) 033026.

[17] D. Zhang, M.N. Slipchenko, J.X. Cheng, The Journal of Physical Chemistry Letters, 2 (2011) 1248-1253.

[18] Y. Ozeki, F. Dake, S. Kajiyama, K. Fukui, K. Itoh, Opt. Express, 17 (2009) 3651-3658.

[19] R. Ellis, E. Green, C.P. Winlove, Connective Tissue Research, 50 (2009) 29-36.

[20] H.W. Wang, T.T. Le, J.X. Cheng, Opt. Commun., 281 (2008) 1813-1822.

[21] A. Pliss, A.N. Kuzmin, A.V. Kachynski, P.N. Prasad, Proc. Natl. Acad. Sci. U. S. A., 107 (2010) 12771-12776.

[22] R. Bansil, I.V. Yannas, H.E. Stanley, Biochimica et Biophysica Acta (BBA) - General Subjects, 541 (1978) 535-542.

[23] B.G. Frushour, J.L. Koenig, biopolymers, 14 (1975) 379-391.

[24] D. Akimov, S. Chatzipapadopoulos, T. Meyer, N. Tarcea, B. Dietzek, M. Schmitt, J. Popp, Journal of Raman Spectroscopy, 40 (2009) 941-947.

[25] S. Lu, W. Min, S. Chong, G.R. Holtom, X.S. Xie, Appl. Phys. Lett., 96 (2010) 113701.

[26] J. Moger, N.L. Garrett, D. Begley, L. Mihoreanu, A. Lalatsa, M.V. Lozano, M. Mazza, A. Schatzlein, I. Uchegbu, Journal of Raman Spectroscopy, (2012) n/a-n/a.

[27] D. Fu, T. Ye, T.E. Matthews, B.J. Chen, G. Yurtserver, W.S. Warren, Opt. Lett., 32 (2007) 2641-2643.

[28] T. Ye, D. Fu, W.S. Warren, Photochemistry and Photobiology, 85 (2009) 631-645.

[29] F. Ghadially, G. Meachim, D. Collins, Annals of the Rheumatic Diseases, 24 (1965) 136.

[30] R. Snyder, S. Hsu, S. Krimm, Spectrochimica Acta Part A: Molecular Spectroscopy, 34 (1978) 395-406.

[31] H.W. Wu, J.V. Volponi, A.E. Oliver, A.N. Parikh, B.A. Simmons, S. Singh, Proc. Natl. Acad. Sci. U. S. A., 108 (2011) 38093814. 
[32] T.T. Le, H.M. Duren, M.N. Slipchenko, C.D. Hu, J.X. Cheng, Journal of Lipid Research, 51 (2010) 672-677.

[33] L. Lippiello, T. Walsh, M. Fienhold, Metabolism, 40 (1991) 571-576.

[34] X. Zhang, M.B.J. Roeffaers, S. Basu, J.R. Daniele, D. Fu, C.W. Freudiger, G.R. Holtom, X.S. Xie, Chemphyschem, (2012).

[35] C.P. Pfeffer, B.R. Olsen, F. Ganikhanov, F. Légaré, J. Struct. Biol., 164 (2008) 140-145.

[36] I. Freund, M. Deutsch, A. Sprecher, Biophys. J., 50 (1986) 693-712.

[37] R.M. Williams, W.R. Zipfel, W.W. Webb, Biophys. J., 88 (2005) 1377-1386.

[38] P. Stoller, P.M. Celliers, K.M. Reiser, A.M. Rubenchik, Appl. Optics, 42 (2003) 5209-5219.

[39] C. Rey, M. Shimizu, B. Collins, M. Glimcher, Calcified tissue international, 49 (1991) 383-388. 